Using the Encyclopédie. Ways of knowing, ways of reading. Edited by Daniel Brewer and Julie CandlerHayes

\title{
Geza Szász
}

\section{CpenEdition}

1 Journals

\section{Édition électronique}

URL : https://journals.openedition.org/studifrancesi/40702

DOI : $10.4000 /$ studifrancesi.40702

ISSN : 2421-5856

\section{Éditeur}

Rosenberg \& Sellier

\section{Édition imprimée}

Date de publication : 1 juillet 2004

Pagination : 191-193

ISSN : 0039-2944

\section{Référence électronique}

Geza Szász, "Using the Encyclopédie. Ways of knowing, ways of reading. Edited by Daniel Brewer and Julie Candler-Hayes », Studi Francesi [En ligne], 142 (XLVIII | I) | 2004, mis en ligne le 30 novembre 2015, consulté le 09 septembre 2021. URL : http://journals.openedition.org/studifrancesi/40702 ; DOI : https://doi.org/10.4000/studifrancesi.40702

Ce document a été généré automatiquement le 9 septembre 2021.

\section{cc) (†) $\odot$}

Studi Francesi è distribuita con Licenza Creative Commons Attribuzione - Non commerciale - Non opere derivate 4.0 Internazionale. 


\title{
Using the Encyclopédie. Ways of knowing, ways of reading. Edited by Daniel Brewer and Julie Candler- Hayes
}

\author{
Geza Szász
}

\section{RÉFÉRENCE}

Using the Encyclopédie. Ways of knowing, ways of reading. Edited by Daniel BREWER and Julie CANDLER-HAYES, Oxford, Voltaire Foundation, 2002, «Studies on Voltaire and the Eighteenth Century», 2002:05), pp. X1+289.

1 Quinze études en anglais ou en français essaient de reconstruire les réseaux du savoir et de la compréhension établis par l'Encyclopédie. De l'aveu des éditeurs (Preface or dialogue between A and B, pp. I-XI), l'idée d'un tel recueil est venue de Tony Strugnell. Ceci dans le contexte des changements profonds de la recherche: la version électronique offre une lecture transversale, l'étude des renvois et des juxtapositions sur l'ensemble de l'oeuvre.

2 Deux principales méthodes d'analyse se dégagent des études. On examine soit des sujets particuliers, soit l'ordre encyclopédique ou l'épistémologie de la lecture. Elles convergent cependant au même but, aider le lecteur du XXI siècle à comprendre comment les encyclopédistes considéraient leur entreprise, et aussi à lire et à connaître l'ouvrage. Cet objectif dépasse évidemment les seuls textes de l'Encyclopédie: on reçoit beaucoup de renseignements sur le monde des Lumières aussi.

David BATES (Cartographic aberrations: epistemology and order in the encyclopedic map, pp. 1-20) retrouve le véritable ordre de l'Encyclopédie dans la métaphore de la mappemonde. Il démontre la complexité de la figure des plans qui offrent une voie pour la compréhension. Le texte encyclopédique constitue une carte qui rend possible la 
découverte des liens cachés; mais elle restera incomplète, puisqu'il y aura toujours des domaines inexplorés dans la pensée humaine.

Daniel BREWER (Constructing philosophers, pp. 21-35) définit les relations des encyclopédistes au savoir, et étudie une des images fondatrices de l'encyclopédisme. L'examen de l'évolution du terme et de la figure du philosophe nous aide à comprendre comment l'Encyclopédie fournissait des idées aux rédacteurs et aux lecteurs lors de la construction d'un sujet «moderne».

5 Fabienne-Sophie CHAUDERLOT (Encyclopédismes d'hier et d'aujourd'hui: information ou pensée? Une lecture de l'Encyclopédie à la Deleuze, pp. 37-62) propose un autre type de lecture, rompt avec la vision de l'Encyclopédie en tant que trésor monolithique des connaissances, et souligne la contribution de l'informatisation à la modification des concepts. On peut désormais traiter l'Encyclopédie comme le livre rhizome de Deleuze et Guattari et réaliser toutes les possibilités de sa «textualité multiple».

6 Patrick COLEMAN ('Figure' in the Encyclopédie: discovery or discipline, pp. 63-79) étudie les modalités de la lecture utilisées au milieu du XVIII ${ }^{\mathrm{e}}$ siècle. L'examen des articles de l'Encyclopédie reflétant la pratique de la lecture des éditeurs mêmes pourrait aider à découvrir comment on pouvait ou devait lire l'Encyclopédie pendant les années 1750.

7 Thomas DIPIERo (Bodies of knowledge, pp. 81-97) nous découvre que les auteurs de l'Encyclopédie ont considéré le corps humain à la fois comme objet et comme moyen de connaissance. Selon son constat, l'homme blanc représentait la beauté, alors que le blanc était la «couleur naturelle» de l'«humaine espece».

8 Julie CANDLER-HAYES (Translation (in)version and the encyclopédic network, pp. 99-118) retrouve, malgré le silence de l'Encyclopédie, tous les points essentiels du débat sur la traduction du XVIII ${ }^{e}$ siècle, et analyse les conceptions sur la langue, «l'ordre naturel», la distinction entre traduction et version aussi bien que l'évolution de la figure du traducteur.

9 Cynthia J. KOEPP (Making money: artisans and entrepreneurs in Diderot's Encyclopédie, pp. 119-141) rectifie un des lieux communs de la recherche, la «célébration de l'artisan». Elle évoque les ambiguïtés des articles, les préjugés ou bien les frustrations linguistiques. Un autre aspect de l'Encyclopédie est également étudié: il s'y agit en fait d'un manuel de management et d'économie (libérale) des Lumières.

Robert MORISSEY (The Encyclopédie: monument for a nation, pp. 143-161) analyse le paradoxe de la coexistence du nationalisme et des valeurs universelles promues par les philosophes. Grâce à la version électronique, on découvre, malgré un ouvrage assez tacite sur le sujet, que la France est le pays le plus mentionné, alors que nation se retrouve 3646 fois à travers le texte. On pourra alors définir les acceptations des termes à l'époque des Lumières.

11 Pierre SAINT-AMAND (Les progrès de la civilité dans l'Encyclopédie, pp. 163-171) étudie la manière dont la hiérarchie sociale était pensée dans l'Encyclopédie et nous fait voir les efforts de donner un fondement anthropologique au terme de la civilité, aussi bien que l'idée selon laquelle seul le mérite pourrait exiger le respect. L'analyse des articles relatifs à la sociabilité montre la mise en question de l'ancien ordre des considérations. Philip STEWART (The Encyclopédie on-line, pp. 173-185) considère l'ampleur du changement entre le XVIII ${ }^{e}$ siècle et le $\mathrm{XXI}^{\mathrm{e}}$, et énumère les traits auxquels tout lecteur et/ou chercheur de nos jours devrait faire attention pour que la version on-line puisse maintenir les valeurs de l'Encyclopédie. Outil d'une consultation rapide et facile, la 
version électronique nous offre l'ensemble de l'ouvrage et des renvois, but inaccessible pour les rédacteurs. Mais elle risque de devenir une gigantesque pile de mots, au lieu d'une base de données de l'évolution intellectuelle qu'était l'Encyclopédie du XVIII ${ }^{\mathrm{e}}$ siècle.

Downing A. THомAs (Taste, commonality and musical imagination in the Encyclopédie, pp. 187-209) s'occupe des questions du goût, de la communauté et de l'imagination musicale, relevant plutôt de l'irrationnel que de la raison «encyclopédique». Il révèle la fascination des Lumières pour l'irrationnel et l'involontaire, et l'évolution de la considération de l'essence des arts et de leur perception par les hommes. La deuxième partie de l'article démontre l'évolution de la réflexion sur la musique, liée, elle aussi, à la question du goût.

-Marie THORNTON (Translating the garden: references to Philip Miller's The Gardener's dictionary in the Encyclopédie of Diderot and D'Alembert, pp. 211-228) étudie les articles se référant à l'horticulteur anglais Philip Miller, et jette une lumière nouvelle sur la réception de son livre en France. Celle-ci est valeur d'exemple du rôle du matériel de source (et de la traduction «directe») dans la construction et la communication du savoir.

Janie VANPEE (La Femme mode d'emploi: how to read the article FEMME in the Encyclopédie, pp. 229-245) se base sur l'examen de l'article FEMME et de ses renvois sur un des principaux éléments de l'esprit encyclopédique: les renvois pourraient nous renseigner sur les conceptions dominantes d'une époque par rapport à un sujet. FEMME est soit subordonnée à l'HoмmE, soit définie sous les aspects sexuel, conjugal et physique, cette perspective restreinte ne faisant pas sortir l'ouvrage des cadres de la normalité de l'époque.

Anne C. VILA (The body in crisis: vitalisme, hydrotherapy and medical discourse in the Encyclopédie, pp. 246-263) démontre l'influence de la médecine sur les débats philosophiques aussi bien que ses aspirations philosophiques. On ne s'étonne plus que la médecine (de Montpellier) soit un sujet central de l'Encyclopédie. L'école de Montpellier serait aussi à l'origine de la renaissance de l'hydrothérapie et de sa représentation.

Stephen WERNER (The Encyclopédie 'index', pp. 265-270) étudie à la fois structure et lecture de l'Encyclopédie. La démonstration de l'inutilité de toute table analytique aboutit à l'explication du changement opéré par le système référentiel. Selon lui, la version électronique, guidant le lecteur par renvois dans l'ensemble de l'ouvrage, pourrait rendre à l'Encyclopédie l'usage et la lecture que ses éditeurs ont peut-être prévus.

18 La lecture des quinze études nous donne l'impression d'un double hommage. Hommage à l'Encyclopédie, cette «autoroute de l'information du XVIII siècle»; et hommage à la version électronique, qui peut désormais mettre le lecteur plus près des auteurs. Ces derniers n'auraient pas pu demander mieux. 